\title{
High-Order Energy Balance Method to Nonlinear Oscillators
}

\author{
Seher Durmaz and Metin Orhan Kaya \\ Faculty of Aeronautics and Astronautics, Istanbul Technical University, Maslak, 34469 Istanbul, Turkey \\ Correspondence should be addressed to Seher Durmaz, durmazseh@itu.edu.tr
}

Received 1 April 2012; Revised 25 June 2012; Accepted 9 July 2012

Academic Editor: Livija Cveticanin

Copyright (C) 2012 S. Durmaz and M. O. Kaya. This is an open access article distributed under the Creative Commons Attribution License, which permits unrestricted use, distribution, and reproduction in any medium, provided the original work is properly cited.

Energy balance method (EBM) is extended for high-order nonlinear oscillators. To illustrate the effectiveness of the method, a cubic-quintic Duffing oscillator was chosen. The maximum relative errors of the frequencies of the oscillator read $1.25 \%$ and $0.6 \%$ for the first- and second-order approximation, respectively. The third-order approximation has an accuracy as high as $0.008 \%$. Excellent agreement of the approximated frequencies and periodic solutions with the exact ones is demonstrated for several values of parameters of the oscillator.

\section{Introduction}

A great deal of work has been devoted to the nonlinear problems encountered in the fields of applied mathematics, physics, and engineering sciences. In general, the analytical approximation to the solution of a given nonlinear problem is difficult, sometimes impossible; plenty of techniques based on numerical methods have been implemented. Among them are the variational iteration method [1,2], the harmonic balance method [3,4], and energy balance method [5-8] developed to solve nonlinear differential equations. In this study, we have investigated the application of high-order energy balance method to cubic-quintic Duffing oscillator. The nonlinear frequencies are calculated for the first-, second-, and thirdorder EBM and compared with the results of different techniques.

\section{The Basic Idea of Energy Balance Method}

This section briefly introduces energy balance method proposed by He [5]. In this method, a variational principle for the oscillation is established, then the corresponding Hamiltonian is considered from which the angular frequency can be easily obtained by Galerkin method. 
Let us consider the motion of a general oscillator with the initial conditions in the form

$$
u^{\prime \prime}+f(u)=0, \quad u(0)=A, \quad u^{\prime}(0)=0,
$$

where $A$ is the initial amplitude.

Its variational can be written as

$$
J(u)=\int_{0}^{T / 4}\left[-\frac{1}{2} u^{\prime 2}+F(u)\right] d t .
$$

Here $T=2 \pi / \omega$ is the period of the nonlinear oscillation and $F(u)=\int f(u) d u$.

The Hamiltonian of (2.1) can be written in the form:

$$
H(u)=\frac{1}{2} u^{\prime 2}+F(u)
$$

In (2.2) the kinetic energy $(E)$ and potential energy $(P)$ can be, respectively, expressed as $E=(1 / 2) u^{\prime 2}, P=F(u)$.

Throughout the oscillation since the system is conservative, the total energy remains unchanged during the motion; the Hamiltonian of the oscillator becomes a constant value,

$$
H=E+T=F(A) .
$$

For the first-order approximation, the following trial function can be assumed:

$$
u(t)=A \cos \omega t
$$

Substituting (2.5) into (2.3) yields the following residual:

$$
R(t)=\frac{A^{2}}{2} \omega^{2} \sin ^{2} \omega t+F(A \cos \omega t)-F(A)
$$

The residual is forced to zero, in an average sense, by setting weighted integrals of the residual to zero

$$
\int_{0}^{T / 4} R(t) w_{n} d t=0, \quad n=1,2, \ldots
$$

where $w_{n}$ is a set of weighting function (or test).

There are lots of weighting functions, that is, Galerkin, least squares, collocation and so forth. In this study, we used Galerkin method as a weighting function. 


\section{High-Order Energy Balance Method}

In order to extend He's energy balance method, let us assume that the solution of (2.1) can be expressed as

$$
u=A_{1} \cos \omega t+A_{2} \cos 3 \omega t+\cdots+A_{n} \cos (2 n-1) \omega t
$$

From the initial conditions, the coefficients should satisfy the following constrain:

$$
A=A_{1}+A_{2}+\cdots+A_{n}
$$

One of these parameters can be chosen as a dependent parameter. Hence,

$$
A_{n}=A-A_{1}-A_{2}-\cdots-A_{n-1} .
$$

By inserting (3.1) into (2.7), the following systems can be obtained:

$$
\begin{gathered}
\int_{0}^{T / 4} R(t) \cos \omega t d t=0, \\
\int_{0}^{T / 4} R(t) \cos 3 \omega t d t=0 \\
\vdots \\
\int_{0}^{T / 4} R(t) \cos (2 n-1) \omega t d t=0 .
\end{gathered}
$$

\section{Example}

A cubic-quintic Duffing oscillator is considered. In the following sections, the nonlinear frequencies will be compared with the results of different techniques to illustrate the efficiency and accuracy of energy balance method.

The governing differential equation of this oscillator is in the form of

$$
u^{\prime \prime}+\alpha u+\varepsilon u^{3}+\lambda u^{5}=0, \quad \text { where } \alpha \geq 0
$$

with the initial conditions

$$
u(0)=A, \quad u^{\prime}(0)=0 .
$$


The Hamiltonian of (4.1a) is given as follows:

$$
H(u)=\frac{1}{2} u^{\prime 2}+\frac{1}{2} u^{2}+\frac{1}{4} \varepsilon u^{4}+\frac{1}{6} \lambda u^{6}=\frac{1}{2} \alpha A^{2}+\frac{1}{4} \varepsilon A^{4}+\frac{1}{6} \lambda A^{6} .
$$

For the first-order approximation, assume that $u(t)$ is in the following form:

$$
u(t)=A \cos \omega t
$$

Substituting the first approximation into (4.2) yields

$$
\begin{aligned}
R_{1}(t)= & \frac{1}{2} A^{2} \omega^{2} \sin ^{2}(\omega t)+\frac{1}{4} \varepsilon A^{4} \cos ^{4}(\omega t)+\frac{1}{6} \lambda A^{6} \cos ^{6}(\omega t)+\frac{1}{2} \alpha A^{2} \cos ^{2}(\omega t) \\
& -\frac{\varepsilon A^{4}}{4}-\frac{\alpha A^{2}}{2}-\frac{\lambda A^{6}}{4}
\end{aligned}
$$

First-order approximation can be obtained by setting

$$
\int_{0}^{T / 4} R_{1}(t) \cos (\omega t) d t=0, \quad T=\frac{2 \pi}{\omega}
$$

The amplitude-frequency relationship for the first approximation is obtained as

$$
\omega(A)=\sqrt{\frac{70 \alpha+49 \varepsilon A^{2}+38 \lambda A^{4}}{70}} .
$$

To obtain a more accurate result, let us define $u$ as follows:

$$
u(t)=A_{1} \cos \omega t+\left(A-A_{1}\right) \cos 3 \omega t
$$

Substituting (4.7) into (4.2) results in the following residual:

$$
\begin{aligned}
R_{2}(t)= & \frac{\omega^{2}}{2}\left[A_{1} \sin (\omega t)+3\left(A-A_{1}\right) \sin (3 \omega t)\right]^{2}+\frac{\alpha}{2}\left[A_{1} \cos (\omega t)+\left(A-A_{1}\right) \cos (3 \omega t)\right]^{2} \\
& +\frac{\varepsilon}{4}\left[A_{1} \cos (\omega t)+\left(A-A_{1}\right) \cos (3 \omega t)\right]^{4}+\frac{\lambda}{6}\left[A_{1} \cos (\omega t)+\left(A-A_{1}\right) \cos (3 \omega t)\right]^{6} \\
& -\frac{\alpha A^{2}}{2}-\frac{\varepsilon A^{4}}{4}-\frac{\lambda A^{6}}{6}
\end{aligned}
$$


Table 1: Comparison of the first-, second-, and third-order frequencies for $\alpha=1$ and $\lambda=0$.

\begin{tabular}{lcccccc}
\hline$\varepsilon A^{2}$ & $\omega_{\text {Exact }}$ & $\omega_{1 \text {-EBM }}$ & $\omega_{2 \text {-EBM }}$ & $\omega_{3 \text {-EBM }}$ & {$[4]^{*}$} & {$[9]^{*}$} \\
\hline 0.5 & 1.17078 & 1.16190 & 1.17026 & 1.17078 & 1.17078 & 1.17078 \\
1 & 1.31778 & 1.30384 & 1.31635 & 1.31776 & 1.31778 & 1.31778 \\
5 & 2.15042 & 2.12132 & 2.14260 & 2.15030 & 2.15047 & 2.15050 \\
10 & 2.86664 & 2.82843 & 2.85355 & 2.86645 & 2.86676 & 2.86683 \\
100 & 8.53359 & 8.42615 & 8.48429 & 8.53290 & 8.53415 & 8.53454 \\
1000 & 26.8107 & 26.4764 & 26.6519 & 26.8085 & 26.8126 & 26.8139 \\
5000 & 59.9157 & 59.1692 & 59.5599 & 59.9108 & 59.9198 & 59.9229 \\
\hline
\end{tabular}

*Third-order approximations.

We set

$$
\begin{aligned}
\int_{0}^{T / 4} R_{2}(t) \cos (\omega t) d t= & -6550410 \lambda A^{6}-14096128 \lambda A^{5} A_{1}+A^{4}\left(-8898327 \varepsilon+59159040 \lambda A_{1}^{2}\right) \\
& -128 A^{3}\left(131461 \varepsilon A_{1}+979520 \lambda A_{1}^{3}\right) \\
& +32 A_{1}^{2}\left(831402 \alpha+723520 A_{1}^{2} \varepsilon+946176 \lambda A_{1}^{4}+3187041 \omega^{2}\right) \\
& -48 A A_{1}\left(461890 \alpha+1033600 A_{1}^{2} \varepsilon+2150400 \lambda A_{1}^{4}+4572711 \omega^{2}\right) \\
& +2 A^{2}\left(-7066917 \alpha+22697856 A_{1}^{2} \varepsilon+77271040 \lambda A_{1}^{4}+63602253 \omega^{2}\right)=0
\end{aligned}
$$

$$
\begin{aligned}
\int_{0}^{T / 4} R_{2}(t) \cos (3 \omega t) d t= & 5265546 \lambda A^{6}+83291904 \lambda A^{5} A_{1}+A^{4}\left(6789783 \varepsilon-313950720 \lambda A_{1}^{2}\right) \\
& +2176 A^{3}\left(45429 \varepsilon A_{1}+301120 \lambda A_{1}^{3}\right) \\
& +6 A^{2}\left(1616615 \alpha-37333632 \varepsilon A_{1}^{2}-128921600 \lambda A_{1}^{4}-14549535 \omega^{2}\right) \\
& -96 A_{1}^{2}\left(1016158 \alpha+1012928 \varepsilon A_{1}^{2}+1396736 \lambda A_{1}^{4}+1801371 \omega^{2}\right) \\
& +48 A A_{1}\left(2678962 \alpha+5002624 \varepsilon A_{1}^{2}+10309632 \lambda A_{1}^{4}+4572711 \omega^{2}\right)=0 .
\end{aligned}
$$

By solving (4.9a)-(4.9b) simultaneously, one can obtain the second-order approximate amplitude-frequency relation. For different $\varepsilon A^{2}$ values, the approximate frequencies are given in Table 1.

Moreover, the accuracy of results will be further improved by defining $u$ in the following form:

$$
u(t)=A_{1} \cos \omega t+A_{2} \cos 3 \omega t+\left(A-A_{1}-A_{2}\right) \cos 5 \omega t
$$


Table 2: Comparison of the first-, second-, and third-order frequencies for $\alpha=1, \varepsilon=5$.

\begin{tabular}{lcccc}
\hline$\lambda A^{6}$ & $\omega_{\mathrm{R}-\mathrm{K}}$ & $\omega_{1 \text {-EBM }}$ & {$[4]^{*}$} & $\omega_{3 \text {-EBM }}$ \\
\hline 1 & 2.2798 & 2.2456 & 2.3184 & 2.27948 \\
5 & 2.7318 & 2.6859 & 2.8062 & 2.73144 \\
10 & 3.2057 & 3.1510 & 3.3166 & 3.20446 \\
100 & 7.7762 & 7.6672 & 8.2006 & 7.77554 \\
1000 & 23.7999 & 23.3957 & 25.0948 & 23.7045 \\
\hline
\end{tabular}

${ }^{*}$ First-order approximation, $\omega_{\text {Ref. }[4]}=\sqrt{8 \alpha+6 \varepsilon A^{2}+5 \lambda A^{4} / 8}$.

Substituting (4.10) into (4.2), we get the following residual for the third-order approximation:

$$
\begin{aligned}
R_{3}(t)= & \frac{1}{2} \omega^{2}\left[A_{1} \sin (\omega t)+3 A_{2} \sin (3 \omega t)+5\left(A-A_{1}-A_{2}\right) \sin (5 \omega t)\right]^{2} \\
& +\frac{1}{2} \alpha\left[A_{1} \cos (\omega t)+A_{2} \cos (3 \omega t)+\left(A-A_{1}-A_{2}\right) \cos (5 \omega t)\right]^{2} \\
& +\frac{1}{4} \varepsilon\left[A_{1} \cos (\omega t)+A_{2} \cos (3 \omega t)+\left(A-A_{1}-A_{2}\right) \cos (5 \omega t)\right]^{4} \\
& +\frac{1}{6} \lambda\left[A_{1} \cos (\omega t)+A_{2} \cos (3 \omega t)+\left(A-A_{1}-A_{2}\right) \cos (5 \omega t)\right]^{6} \\
& -\frac{\alpha A^{2}}{2}-\frac{\varepsilon A^{4}}{4}-\frac{\lambda A^{6}}{6} .
\end{aligned}
$$

Inserting (4.11) into (3.3) for $n=3$ and using the same procedure explained above, we get three weighted integrals. Solving these three equations simultaneously, the amplitude-frequency relation for the third-order approximation is obtained. For higher-order approximations, the similar procedures can be applied, however, the accuracy of the thirdorder approximation is appropriate for several values of parameters $\alpha, \varepsilon$, and $\lambda$.

In the following, the nonlinear frequencies for the cubic-quintic oscillator are calculated for two different cases: (i) $\lambda=0$ and (ii) $\lambda \neq 0$.

The first case considered here corresponds to the cubic Duffing oscillator. For $\lambda=0$, the results of the nonlinear frequencies are obtained by the first-, second-, and third-order energy balance method and compared with the results of $[4,9]$. In the second case, for $\lambda \neq 0$, the nonlinear frequencies are given in Table 2. Additionally, the numerical solution for all cases is acquired by standard Runge-Kutta method (R-K).

In Table 1, the relative errors for the first-order approximation read $1.25 \%$, while this error reduces to $0.59 \%$ in the second approximation. We observe that the differences between the third-order and the exact frequencies are sufficiently small.

As seen in Table 2, the results of third order approximation are in very good agreement between the numerical results. The comparison of approximate and numerical solutions can also be found in Figure 1. It can be seen that the first-, and the second-order results have slight differences compared to the numerical solution. However, the third-order approximation is overlapping with the numerical solution. 


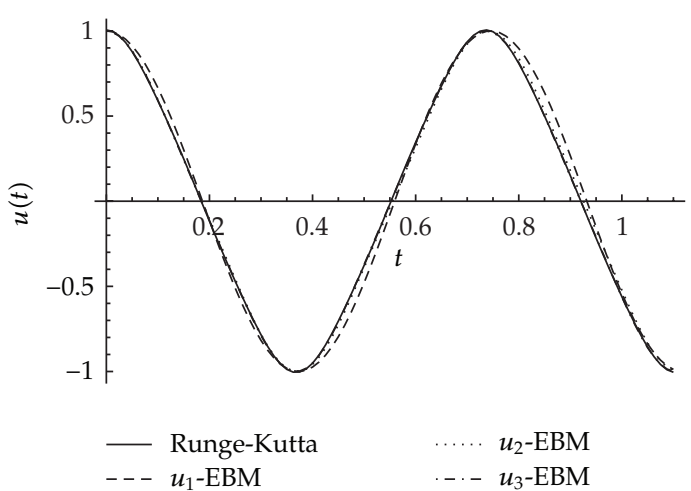

(a)

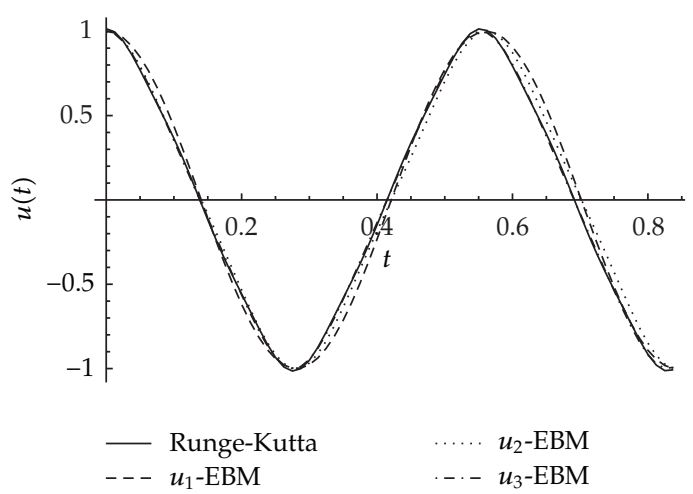

(b)

Figure 1: Approximate and numerical solutions for (a) $A=\alpha=1, \varepsilon=100, \lambda=0$; (b) $A=\alpha=1, \varepsilon=\lambda=$ 100 .

\section{Conclusion}

In this paper, energy balance method is extended for high-order solutions. The first-order approximate frequency for Duffing oscillator gives $1.25 \%$ relative error, while the second-, and the third-order approximated frequencies reach $0.59 \%$ and $0.008 \%$ relative errors, respectively. Moreover, relative errors in high-order energy balance reduce to smaller values than global error minimization and harmonic balance methods. Consequently, we can state that extended method is very effective and convenient for the cubic-quintic Duffing oscillator.

\section{References}

[1] J.-H. He, "Variational iteration method—some recent results and new interpretations," Journal of Computational and Applied Mathematics, vol. 207, no. 1, pp. 3-17, 2007.

[2] V. Marinca and N. Herisanu, "Periodic solutions for some strongly nonlinear oscillations by He's variational iteration method," Computers \& Mathematics with Applications, vol. 54, no. 7-8, pp. 11881196, 2007.

[3] A. Beléndez, E. Gimeno, T. Beléndez, and A. Hernández, "Rational harmonic balance based method for conservative nonlinear oscillators: application to the Duffing equation," Mechanics Research Communications, vol. 36, no. 6, pp. 728-734, 2009.

[4] B. S. Wu, W. P. Sun, and C. W. Lim, "An analytical approximate technique for a class of strongly nonlinear oscillators," International Journal of Non-Linear Mechanics, vol. 41, no. 6-7, pp. 766-774, 2006.

[5] J. H. He, "Preliminary report on the energy balance for nonlinear oscillations," Mechanics Research Communications, vol. 29, no. 2-3, pp. 107-111, 2002.

[6] H.-L. Zhang, "Periodic solutions for some strongly nonlinear oscillations by He's energy balance method," Computers \& Mathematics with Applications, vol. 58, no. 11-12, pp. 2480-2485, 2009.

[7] M. Momeni, N. Jamshidi, A. Barari, and D. D. Ganji, "Application of He's energy balance method to Duffing-harmonic oscillators," International Journal of Computer Mathematics, vol. 88, no. 1, pp. 135-144, 2011.

[8] A. Yildirim, H. Askari, Z. Saadatnia, M. KalamiYazdi, and Y. Khan, "Analysis of nonlinear oscillations of a punctual charge in the electric field of a charged ring via a Hamiltonian approach and the energy balance method," Computers \& Mathematics with Applications, vol. 62, no. 1, pp. 486-490, 2011.

[9] Y. Farzaneh and A. Akbarzadeh Tootoonchi, "Global error minimization method for solving strongly nonlinear oscillator differential equations," Computers \& Mathematics with Applications, vol. 59, no. 8, pp. 2887-2895, 2010. 


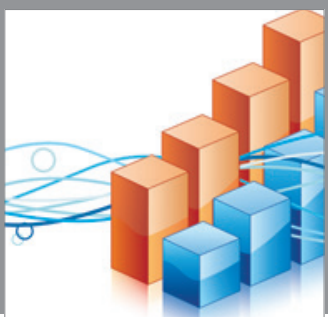

Advances in

Operations Research

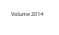

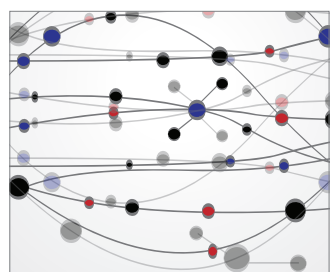

\section{The Scientific} World Journal
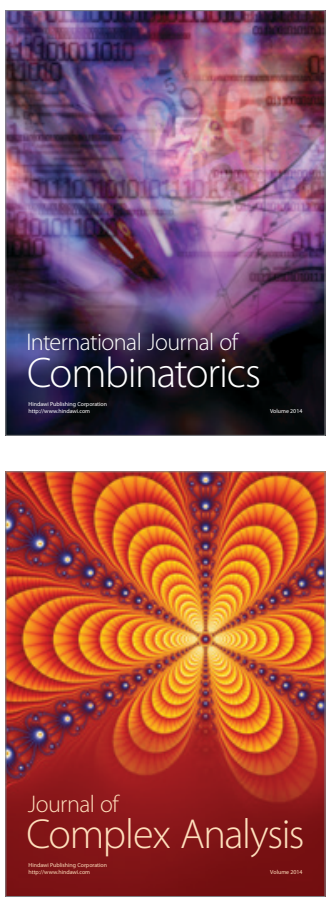

International Journal of

Mathematics and

Mathematical

Sciences
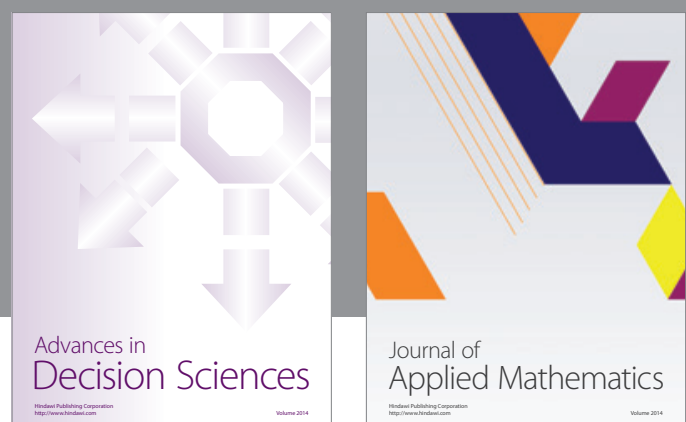

Journal of

Applied Mathematics
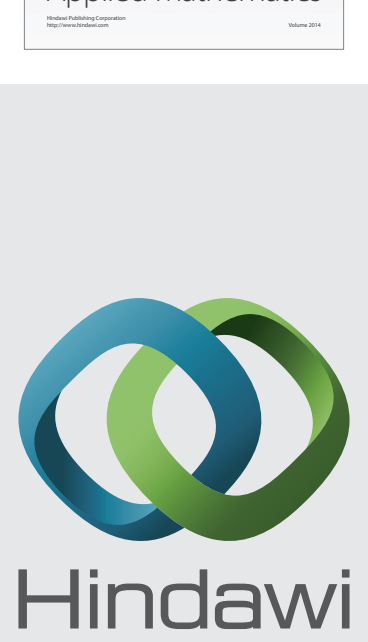

Submit your manuscripts at http://www.hindawi.com
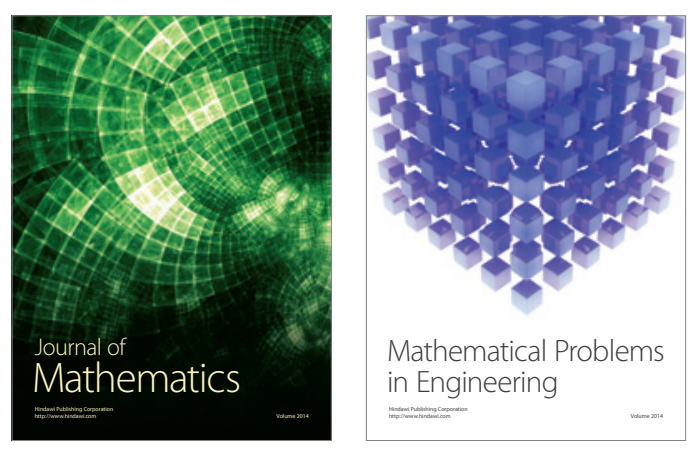

Mathematical Problems in Engineering
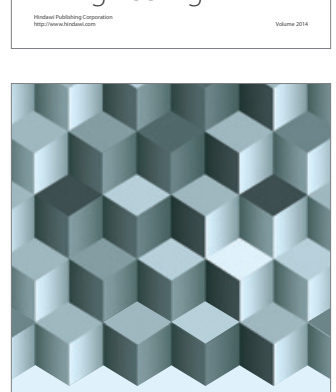

Journal of

Function Spaces
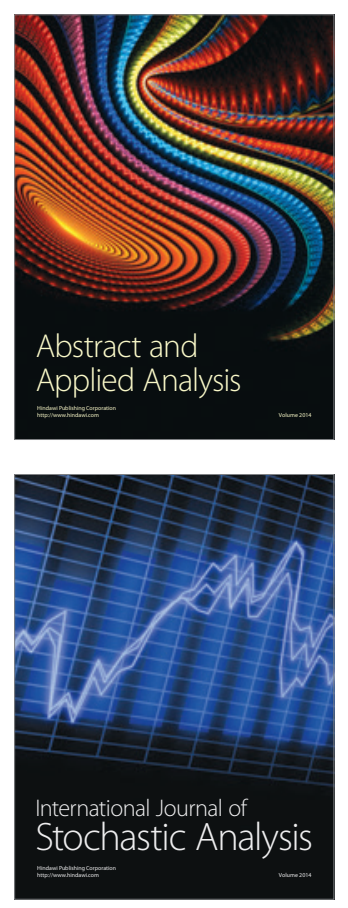

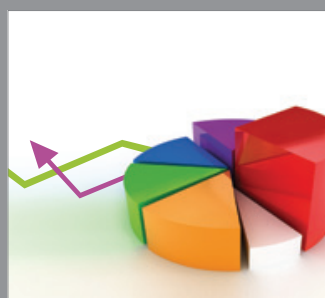

ournal of

Probability and Statistics

Promensencen
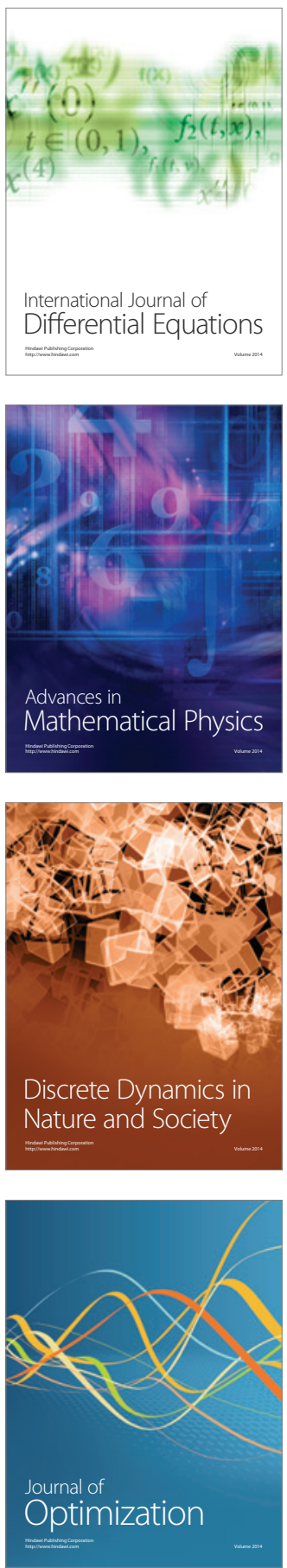